\title{
Connecting Performance Artists with Digital Audiences: A Case Study of Scratch Online
}

\author{
Eric T. Meyer \\ Oxford internet Institute \\ University of Oxford \\ 1 St. Giles \\ Oxford OX1 3SJ \\ United Kingdom \\ eric.meyer@oii.ox.ac.uk
}

\author{
Isis Hjorth \\ Oxford Internet Institute \\ University of Oxford \\ 1 St. Giles \\ Oxford OX1 3SJ \\ United Kingdom \\ isis.hjorth@oii.ox.ac.uk
}

The Battersea Arts Centre (London, UK) has been a pioneer in developing new models of theatre, including creating and supporting the Scratch programme for the last 12 years. At its core, Scratch is about giving artists-in-residence the opportunity to present work-in-progress, and to actively seek feedback from a live audience. Portions of new works are trialled in short pieces often lasting just 15 minutes, followed by discussions and feedback. With new funding from NESTA, the Battersea Arts Centre and several partners are in the process of developing a Scratch Online platform to help commissioned artists extend this engagement with audiences into an online space using digital tools including live and recorded video.

This talk will report on the Scratch Online process from the point of view of an external social science research team which has been following the design, implementation, and artistic uses of Scratch Online from the onset of the project in 2011. The research study focuses on understanding the Scratch Online process' impact on the work of artists, on the cultural institution itself, and asks whether digital modes of work enable engagement with new audiences or in new ways. Drawing on data from semi-structured interviews, data from online sources, and observations of the rehearsal, performance, and feedback process, we present preliminary answers to the following question: When existing non-digital artistic and theatrical production practices are enhanced or replaced with digital tools and techniques, what are the consequences for participation, practice, and behaviour?

The research is jointly funded by the Arts and Humanities Research Council (AHRC), NESTA, and Arts Council England. 